\title{
Comparison of gene expression profiles between dental pulp and periodontal ligament tissues in humans
}

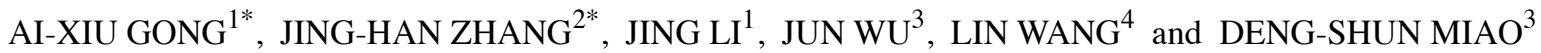 \\ Departments of ${ }^{1}$ Stomatology, and ${ }^{2}$ Neonatology, Children's Hospital of Nanjing Medical University, Nanjing, \\ Jiangsu 210008; ${ }^{3}$ State Key Laboratory of Reproductive Medicine, The Research Center for Bone and Stem Cells, \\ Department of Anatomy, Histology and Embryology, Nanjing Medical University; ${ }^{4}$ Jiangsu Key Laboratory of Oral Diseases, \\ Department of Orthodontics, School of Stomatology, Nanjing Medical University, Nanjing, Jiangsu 210029, P.R. China
}

Received March 16, 2016; Accepted June 16, 2017

DOI: 10.3892/ijmm.2017.3065

\begin{abstract}
There are anatomical and functional differences between human dental pulp (DP) and periodontal ligament (PDL). However, the molecular biological differences and function of these tissues are poorly understood. In the present study, we employed a cDNA microarray array to screen for differentially expressed genes (DEGs) between human DP and PDL tissues, and used the online software WebGestalt to perform the functional analysis of the DEGs. In addition, the STRING database and KEGG pathway analysis were applied for interaction network and pathway analysis of the DEGs. DP and PDL samples were obtained from permanent premolars $(n=16)$ extracted for orthodontic purposes. The results of the microarray assay were confirmed by RT-qPCR. The DEGs were found to be significantly associated with the extracellular matrix and focal adhesion. A total of 10 genes were selected to confirm the results. The mRNA levels of integrin alpha 4 (ITGA4), integrin alpha 8 (ITGA8), neurexin 1 (NRXN1) and contactin 1 (CNTN1) were significantly higher in the DP than in the PDL tissues. However, the levels of collagen type XI alpha 1 (COL11A1), aggrecan (ACAN), collagen type VI alpha 1 (COL6A1), chondroadherin (CHAD), laminin gamma 2 (LAMC2) and laminin alpha 3 (LAMA3) were higher in the PDL than in the
\end{abstract}

Correspondence to: Professor Deng-Shun Miao, State Key Laboratory of Reproductive Medicine, The Research Center for Bone and Stem Cells, Department of Anatomy, Histology, and Embryology, Nanjing Medical University, 136 Hanzhong Road, Nanjing, Jiangsu 210029, P.R. China

E-mail:dsmiao@njmu.edu.cn

Professor Lin Wang, Jiangsu Key Laboratory of Oral Diseases, Department of Orthodontics, School of Stomatology, Nanjing Medical University, 136 Hanzhong Road, Nanjing, Jiangsu 210029, P.R. China E-mail:1w603@njmu.edu.cn

${ }^{*}$ Contributed equally

Key words: dental pulp, periodontal ligament, microarray, differentially expressed genes
DP samples. The gene expression profiles provide novel insight into the characterization of DP and PDL tissues, and contribute to our understanding of the potential molecular mechanisms of dental tissue mineralization and regeneration.

\section{Introduction}

Dental pulp (DP) tissue, termed as the 'ectomesenchyme', is derived from ectodermal cells that grow on the periphery of the neural tube, migrate to the oral position and then differentiate into cells of the mesenchymal phenotype (1). Epithelial cells form ameloblasts and odontoblasts, DP and periodontal ligament (PDL) (2). DP is responsible for the maintenance and repair of the periodontal tissue and its related immune system, and it has a high regenerative potency and responds to various types of damage (3). PDL connects the tooth and the alveolar jaw bone in the area surrounding the root surface, and functions as continuous support, attachment, proprioception and physical protection for the teeth, and minimizes tissue damage arising from trauma and infection (4). Owing to the anatomical and functional differences between human DP and PDL, it is reasonable to assume that there are also differences in the gene expression profiles of these tissues.

Previous studies have indicated that the gene expression patterns of mesenchymal stem cells (MSCs) derived from dental tissues are different from those of other tissue by employing genome-wide gene expression profiling and gene ontology analysis (5). Differentially expressed proteins have also been demonstrated between dental and non-dental ovine MSC populations from the same donor, which may attribute to their unique growth and capacity to generate structures resembling the specific microenvironments from which they were derived in vivo $(6,7)$.

Recent findings suggest that human DP-derived stem cells (DPSCs) and PDL-derived stem cells (PDLSCs) have the ability to regenerate a dentin/pulp-like and cementum/PDL-like complex, respectively when they are transplanted into the subcutaneous space of immunocompromised mice (8-11). Stem cell-based dentistry has emerged as a promising alternative for the development of regenerative therapies, which have unavoidable limitations and the effects of which have not yet been fully determined (12). 
The cDNA microarray technique can provide global profiles of gene expression and facilitate the evaluation of large-scale genes simultaneously. This method has been used in dental studies to compare differentially expressed genes (DEGs) among various types of stem cells (13), or tissues (14-16), or diseases (17-19). However, the differences in gene expression profiles between DP and PDL tissue have not yet been fully elucidated. The use of tissue samples provides more information of the actual situation as the interactions between different cell types can be important for the function of tissues.

Therefore, the present study aimed to evaluate and compare the gene expression patterns in DP and PDL tissues from human permanent teeth, and to identify their molecular biological differences and functions. Furthermore, the results may provide insight into the potential molecular mechanisms of dental tissue regeneration.

\section{Materials and methods}

Gene expression data. A gene expression data set (accession no. GSE50639), which included 3 DP and PDL tissues, was downloaded from the Gene Expression Omnibus (http://www.ncbi.nlm.nih.gov/geo). Gene expression levels were measured through the Affymetrix Human Gene 1.0 ST Array beadchip platform (Affymetrix, Santa Clara, CA, USA) (http://www.ncbi.nlm.nih.gov/geo/query/acc.cgi?acc=GPL6244). Platform annotation files were also acquired.

DP and PDL samples. Tissues were obtained from healthy permanent premolars $(n=16$; from 8 males and 8 females, aged 11-14 years) extracted for orthodontic purposes under approved guidelines set by Nanjing Children's Hospital, Nanjing, China. Written informed consent was obtained from all the respective parents or legal guardians.

The DP and PDL samples used for the experiment were collected according to a previously described procedure $(8,9)$. Briefly, tooth surfaces were cleaned with sterile water, and PDL tissues were gently separated from the middle-third of root with a scalpel. The root was then cut around the cementum-enamel junction using sterilized dental fissure burs, and fractured off along the cutting line with sharp-edged pliers to reveal the pulp chamber. The DP tissues were carefully removed from the crown and root. The extracted PD and PDL samples were then immediately frozen and stored in liquid nitrogen.

Microarray data analysis. The expression data were generated using Affymetrix Expression Console software version 1.4 (Affymetrix). The Robust Multi-array Average (RMA) algorithm implemented through the Affymetrix Expression Console software was used to normalize the data. A one-way ANOVA was performed on the RMA expression values to determine whether genes were differentially expressed between DP and PDL groups. A multiple-testing correction was applied to the $\mathrm{p}$-values of the F-statistics to adjust the false discovery rate (20). Genes with adjusted F-statistic p-values of $<0.05$ were extracted. Microarray analysis identified 1,405 genes with differences in expression of $\geq 2$-fold, 920 and 485 of which were more abundant in the DP and PDL tissue, respectively. However, only strongly expressed genes in the DP or PDL tissue, which differed by $>4$ - or 2.5 -fold from the signal of the DP and PDL tissues, respectively, were selected for further analysis.. In order to classify the co-expression gene group with a similar expression pattern, hierarchical clustering analysis was conducted using Affymetrix Transcriptome Analysis Console (TAC) software. The WEB-based Gene Set AnaLysis Toolkit was performed for the biological interpretation of DEGs $(21,22)$. WebGestalt is a system that facilitates the analysis of sets of genes that can be visualized and organized by a user-selected method. These genes were classified based on data on gene function in the gene ontology of the Kyoto Encyclopedia of Genes and Genomes (KEGG) pathway database. Protein-protein interaction (PPI) networks represents a significant step in the elucidation of the underlying molecular mechanisms. In our study, PPI networks were constructed for the protein products using information from the Search Tool for the Retrieval of Interacting Genes/Proteins (STRING, version 9.1; http://string-db.org/) (23). Interactions with a score (i.e., required confidence) $>0.4$ were retained in the network.

Reverse-transcription-quantitative polymerase chain reaction $(R T-q P C R)$. RNA was isolated from the DP and PDL tissues using TRIzol reagent (Invitrogen, Carlsbad, CA, USA) according to the manufacturer's instructions. To determine the number of cDNA molecules in the reverse-transcribed samples, qPCR analyses were performed using the LightCycler system (Roche, Indianapolis, IN, USA). PCR was conducted using $2 \mu \mathrm{l}$ LightCycler DNA Master SYBR-Green I (Roche), $12.5 \mu \mathrm{l}$ of reaction mixture, $2 \mu \mathrm{l}$ of each $5^{\prime}$ and $3^{\prime}$ primer, $2 \mu \mathrm{l}$ samples. $\mathrm{H}_{2} \mathrm{O}$ was then added to a final volume of $25 \mu \mathrm{l}$. The samples were denatured at $95^{\circ} \mathrm{C}$ for $10 \mathrm{sec}$, with a temperature transition rate of $20^{\circ} \mathrm{C} / \mathrm{sec}$. Four steps were carried out in amplification and fluorescence determination: denaturation at $95^{\circ} \mathrm{C}$ for $1 \mathrm{sec}$, with a temperature transition rate of $20^{\circ} \mathrm{C} / \mathrm{sec}$; annealing for $5 \mathrm{sec}$, with a temperature transition rate of $8^{\circ} \mathrm{C} / \mathrm{sec}$; extension at $72^{\circ} \mathrm{C}$ for $20 \mathrm{sec}$, with a temperature transition rate of $4^{\circ} \mathrm{C} / \mathrm{sec}$; and detection of SYBR-Green fluorescence, which reflects the amount of double-stranded DNA, at $86^{\circ} \mathrm{C}$ for $3 \mathrm{sec}$. The amplification cycle number was 35 . To discriminate specific from non-specific cDNA products, a melting curve was obtained at the end of each run. Products were denatured at $95^{\circ} \mathrm{C}$ for $3 \mathrm{sec}$, and the temperature then decreased to $58^{\circ} \mathrm{C}$ for $15 \mathrm{sec}$ and increased slowly from 58 to $95^{\circ} \mathrm{C}$ using a temperature transition rate of $0.1^{\circ} \mathrm{C} / \mathrm{sec}$. To determine the number of copies of the targeted DNA in the samples, purified PCR fragments of known concentrations were serially diluted and served as external standards that were measured in each experiment. Data were normalized to glyceraldehyde 3 -phosphate dehydrogenase (GAPDH) levels in the samples. The primer sequences used for PCR are listed in Table I.

Statistical analysis. Data from image analysis are presented as the means \pm SEM. Statistical comparisons were made using a two-way ANOVA. A value of $\mathrm{p}<0.05$ was considered to indicate a statistically significant difference.

\section{Results}

Gene expression profiles in human DP and PDL tissue. Affymetrix Transcriptome Analysis Console software was applied to analyze the cDNA microarray data set (GSE50639). 
Table I. Specific primer sequences used for RT-qPCR.

\begin{tabular}{|c|c|c|}
\hline Name & Primers sequences $\left(5^{\prime} \rightarrow 3^{\prime}\right)$ & $\begin{array}{l}\text { Product } \\
\text { size (bp) }\end{array}$ \\
\hline \multirow[t]{2}{*}{ ITGA4 } & F: CACAACACGCTGTTCGGCTA & \\
\hline & R: CGATCCTGCATCTGTAAATCGC & 139 \\
\hline \multirow[t]{2}{*}{ ITGA8 } & F: AGAATGGAGACCTTATTGTGGGA & \\
\hline & R: GAGCCACTTCCGTCTGCTTT & 147 \\
\hline \multirow[t]{2}{*}{ NRXN1 } & F: TAAGTGGCCTCCTAATGACCG & \\
\hline & R: TCGCACCAATACGGCTTCTTT & 91 \\
\hline \multirow[t]{2}{*}{ CNTN1 } & F: CAGCCCTTTCCCGGTTTACAA & \\
\hline & R:TGCTTCTGACCATCCCGTAGT & 170 \\
\hline \multirow[t]{2}{*}{ ACAN } & F: GTGCCTATCAGGACAAGGTCT & \\
\hline & R: GATGCCTTTCACCACGACTTC & 167 \\
\hline \multirow[t]{2}{*}{ COL11A1 } & F: ACCCTCGCATTGACCTTCC & \\
\hline & R: TTTGTGCAAAATCCCGTTGTTT & 128 \\
\hline \multirow[t]{2}{*}{ COL6A1 } & F: ACAGTGACGAGGTGGAGATCA & \\
\hline & R: GATAGCGCAGTCGGTGTAGG & 122 \\
\hline \multirow[t]{2}{*}{ CHAD } & F: CGCGGCCTCAAGCAACTTA & \\
\hline & R: TAGGTCAGCTCGGTCAGGTC & 95 \\
\hline \multirow[t]{2}{*}{ LAMC2 } & F: CAAAGGTTCTCTTAGTGCTCGAT & \\
\hline & R: CACTTGGAGTCTAGCAGTCTCT & 153 \\
\hline \multirow[t]{2}{*}{ LAMA3 } & F: CACCGGGATATTTCGGGAATC & \\
\hline & R: AGCTGTCGCAATCATCACATT & 165 \\
\hline \multirow[t]{2}{*}{ GAPDH } & F: GGAGCGAGATCCCTCCAAAAT & \\
\hline & R: GGCTGTTGTCATACTTCTCATGG & 197 \\
\hline
\end{tabular}

\begin{abstract}
The results indicated that the expression of a total of 1,405 genes was altered by at least 2-fold in one tissue type relative to the other. In the DP tissue, the expression levels of 920 genes were at least double those in the PDL tissues, while in the latter, the expression levels of 485 genes were at least double those in the DP tissue. Ultimately, only strongly expressed genes (529 genes) in the DP or PDL tissue whose expression differed by $>4$ - or 2.5 -fold from the signal of the DP or PDL tissue were evaluated further. In the DP tissue, 255 genes were upregulated by at least 4-fold relative to the PDL tissue, while 274 genes were upregulated by at least 2.5 -fold in the PDL tissue relative to the DP tissue. Hierarchical clustering and a heatmap of the DEGs in the DP and PDL tissue are shown in Fig. 1, and the up- and downregulated genes in the DP tissues (compared with the PDL tissues) are shown in Table II.
\end{abstract}

Gene Ontology (GO) analysis. To analyze the specific biological functions and features of the selected genes, an analysis toolkit (WebGestalt) was applied for GO annotation and enrichment analysis. The DEGs were classified according to biological process (BP), molecular function (MF) or cellular component (CC) using the WebGestalt software package on the basis of hypergeometric tests. The Resulting BP, MF and CC networks are shown as directed acyclic graphs (DAG), which are color-coded (red for p-values <0.05) (Fig. 2A). Biological process enrichment was found for genes associated with cell adhesion, extracellular matrix (ECM) organization, regulation of anatomical structure, morphogenesis and system development. Molecular function enrichment was discovered for genes associated with heparin binding, calcium ion binding, integrin binding and metalloendopeptidase activity. Cellular component enrichment was detected for genes associated with the plasma membrane and ECM. Significantly enriched GO categories under biological process, molecular function and cellular component are indicated in Fig. 2B-D. In the biological process category, the GO terms of biological regulation (257 genes), multicellular organismal process (235 genes), response to stimulus (219 genes), related to metabolic process (211 genes) and developmental process were enriched. In the molecular function category, GO terms related to protein binding (213 genes) and ion binding (167 genes) were enriched. In the cellular component category, GO terms related to membrane (254 genes) were enriched.

Interaction network and pathway analysis of DEGs. To further detect the biological function of the DEGs in the DP and PDL tissue, an interaction network was carried out using STRING 9.1 (23), as shown in Fig. 3A. The interaction network was generated on the basis of experimental and database knowledge. Markov cluster algorithm (MCL) was used to find group associations between these DEGs. The inflation factor was set as 1 on a scale of 1-5.

WebGestalt was used to identify the significantly enriched KEGG pathways. The shared enriched pathways,including ECM-receptor interaction, protein digestion and absorption, focal adhesion and cell adhesion molecules (CAMs) (Fig. 3B and Table III) were determined at the significance levels of $\mathrm{p}<0.05$ in WebGestalt. Among the enriched pathways, the upregulated pathways in the DP group included CAMs and salivary secretion pathways and the downregulated pathways in the DP group included ECM-receptor interaction, protein digestion and absorption and focal adhesion. Compared with GO analysis, KEGG pathway analysis provides biological information in a more detailed and specific manner. Furthermore, good concordance was observed from the STRING interaction network and the enriched functional modules identified by the KEGG and GO enrichment analyses.

Results of RT-qPCR. We selected 10 genes [integrin alpha4 (ITGA4), integrin alpha8 (ITGA8), contactin 1 (CNTN1), neurexin 1 (NRXN1), laminin alpha3 (LAMA3), laminin gamma2 (LAMC2), collagen type XIalpha1 (COL11A1), collagen type VI alpha3 (COL6A3), collagen type VI alpha1 (COL6A1) and chondroadherin (CHAD)] to validate the expression data from microarray analysis using SYBR-Green based RT-qPCR. The expression of these genes was significantly altered in various functional modules, as shown in Table III. The 10 genes with expression levels differing by at least 2-fold between the DP and PDL tissues were selected. The results indicated that the mRNA levels of ITGA4, ITGA8, NRXN1 and CNTN1 were significantly higher in the DP compared 


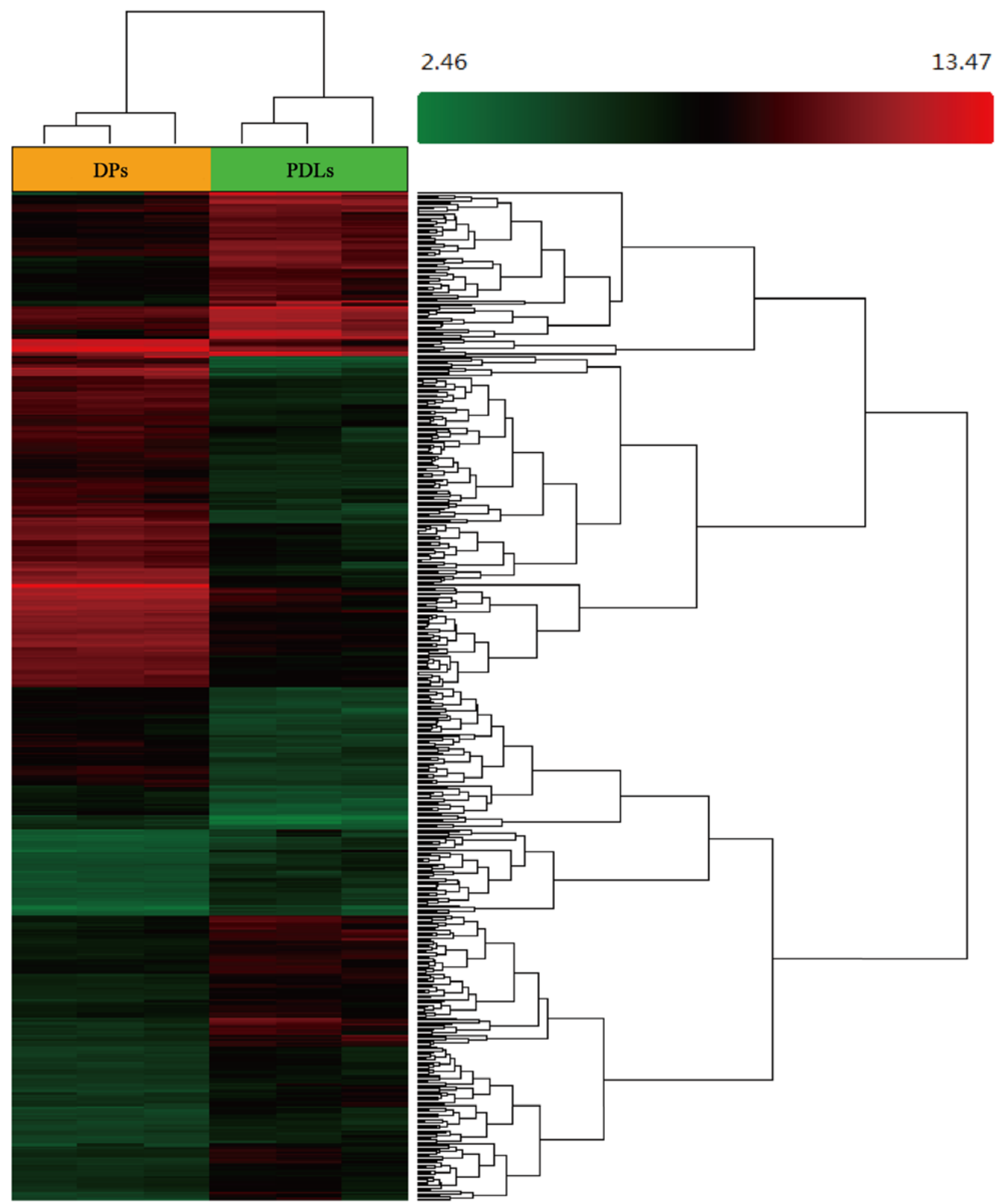

Figure 1. Heatmap of clustering of 1,405 differentially expressed genes in dental pulp (DP) and periodontal ligament (PDL) tissues. Genes expressed with a signal intensity of $>529$ were included in this analysis. Clustering was carried out using Transcriptome Analysis Console (TAC) software (clustering type: hierarchical clustering; distance metric: Pearson's correlation).

with the PDL tissues. However, the levels of COL11A1, ACAN, COL6A1, CHAD, LAMC2 and LAMA3 were higher in the PDL tissue compared with the DP tissue (Fig. 4). The mRNA expression levels demonstrated a consistent trend as the cDNA microarray. Taken together, these results suggested that the two types of tissues have a similar mRNA expression profile, paralleling the results determined by RT-qPCR.

\section{Discussion}

With global gene expression profiling, this study uncovered DEGs from DP and PDL tissues. The cDNA microarray results indicated that the expression levels of 1,405 out of $29,096(4.82 \%)$ genes were altered by at least 2 -fold in one tissue type relative to the other. Lee et al (14) reported that only 

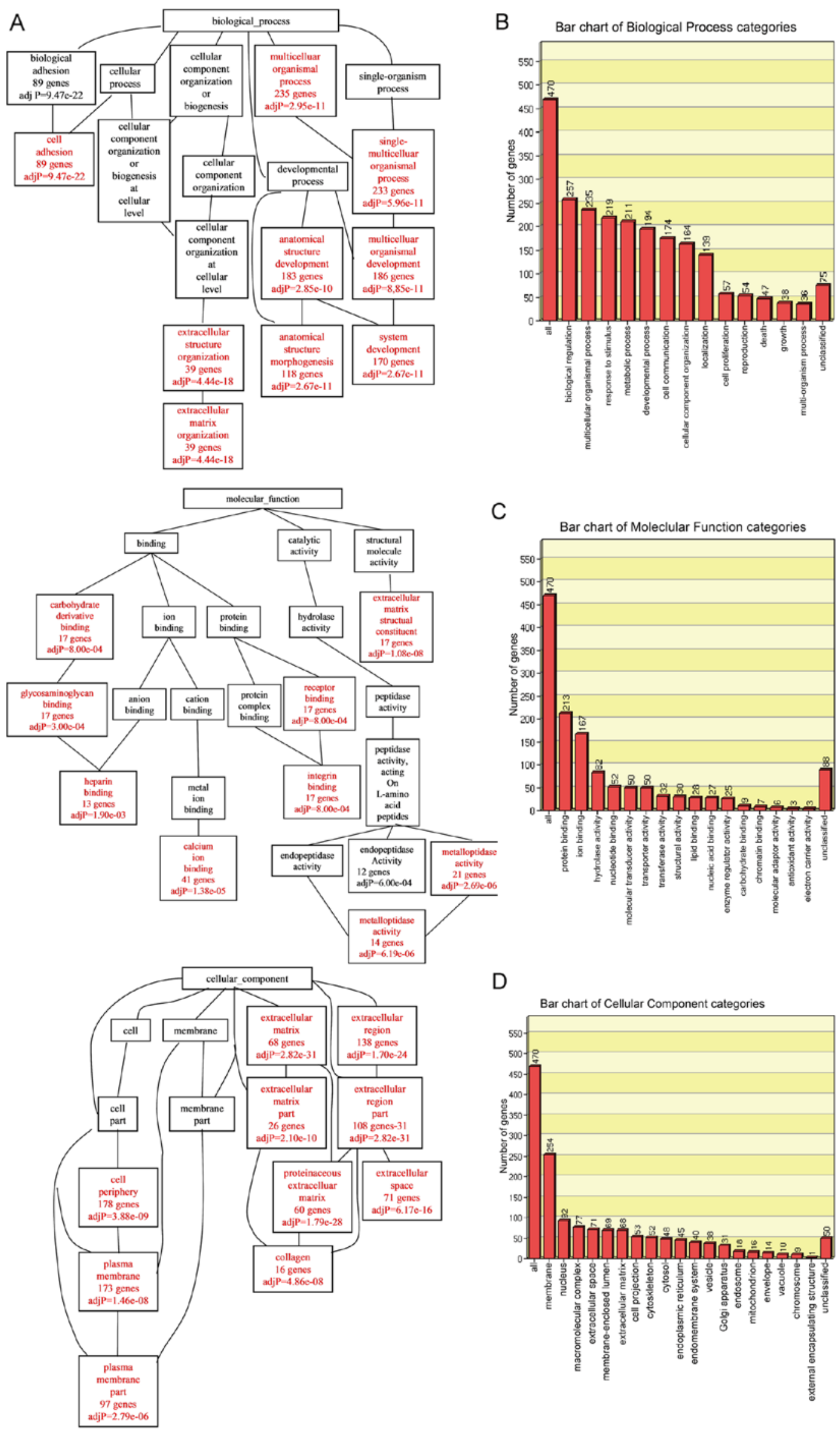

C

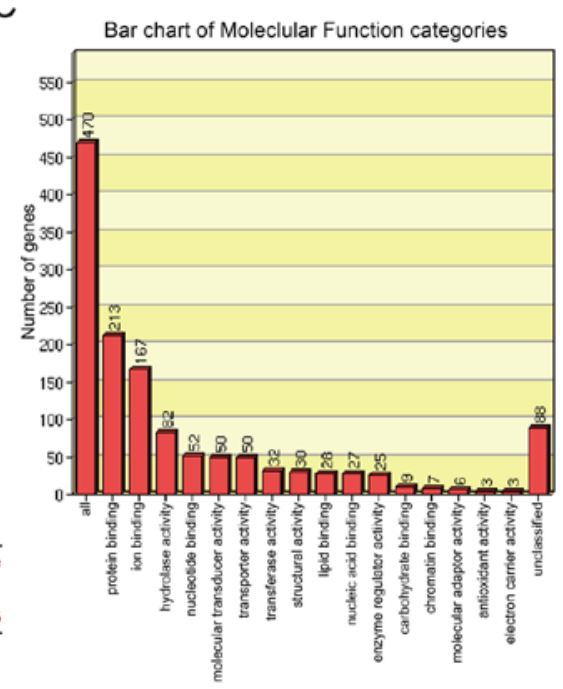

D

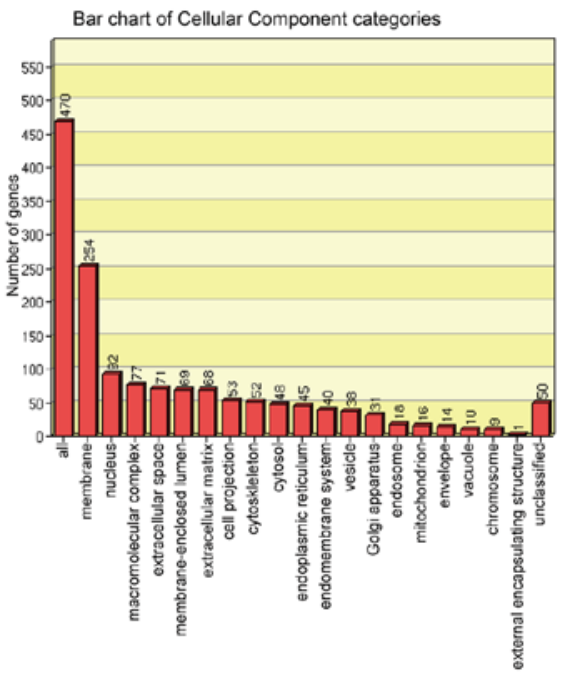

Figure 2. The identified genes were analyzed according to Gene Ontology (GO) enrichment using WebGestalt (WEB-based GEne SeT AnaLysis Toolkit). (A) Directed Acyclic Graph (DAG) of the enriched GO categories under biological process. Each node shows the name of the GO category, the number of gene in the category and the p-value indicating the significance of enrichment. The red colour represents p-values $<0.05$. (B-D) GO classification from the biological process, molecular function and cellular component ontology enrichment. The number of proteins enriched in each GO term is shown on the top of each bar. 


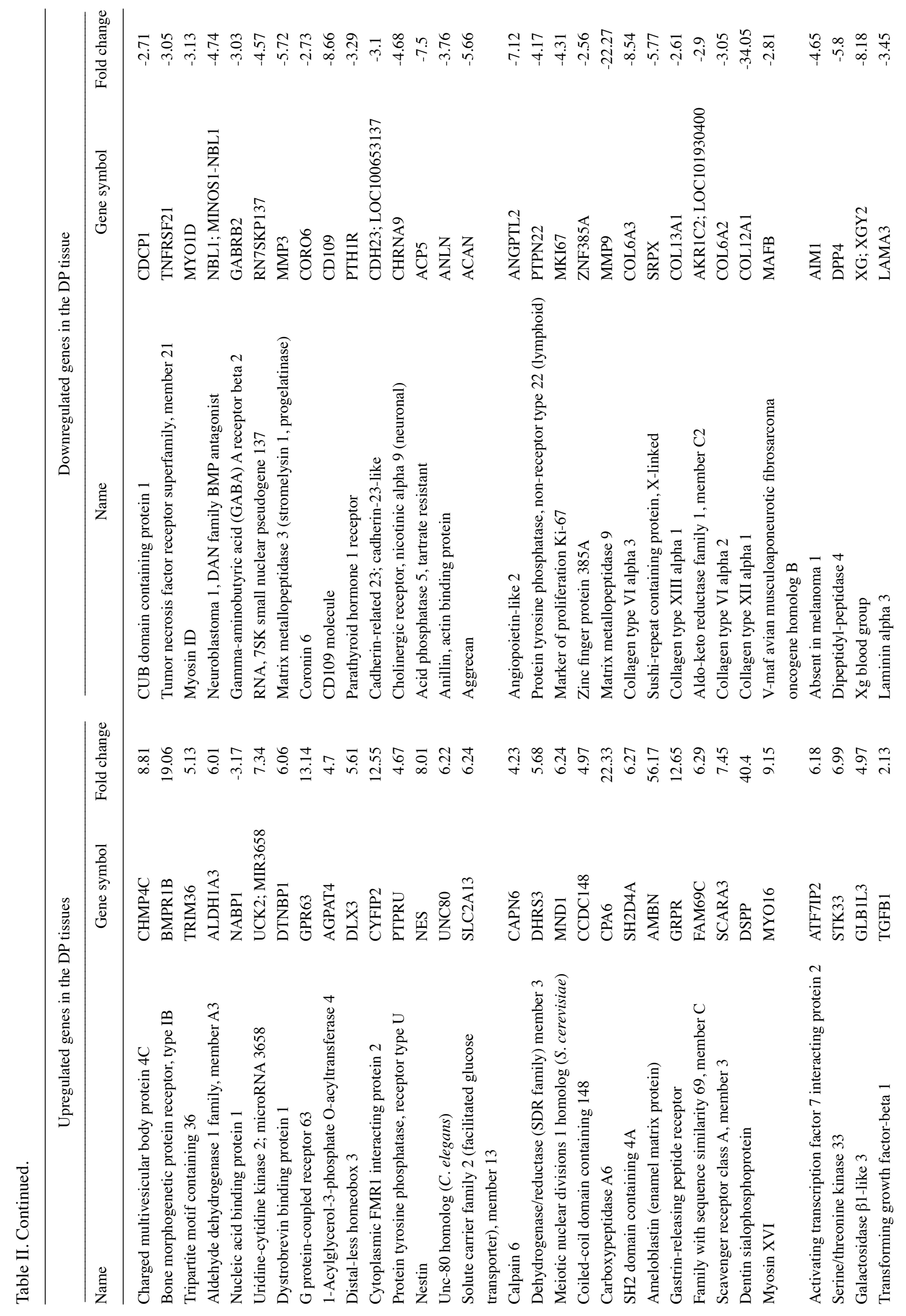




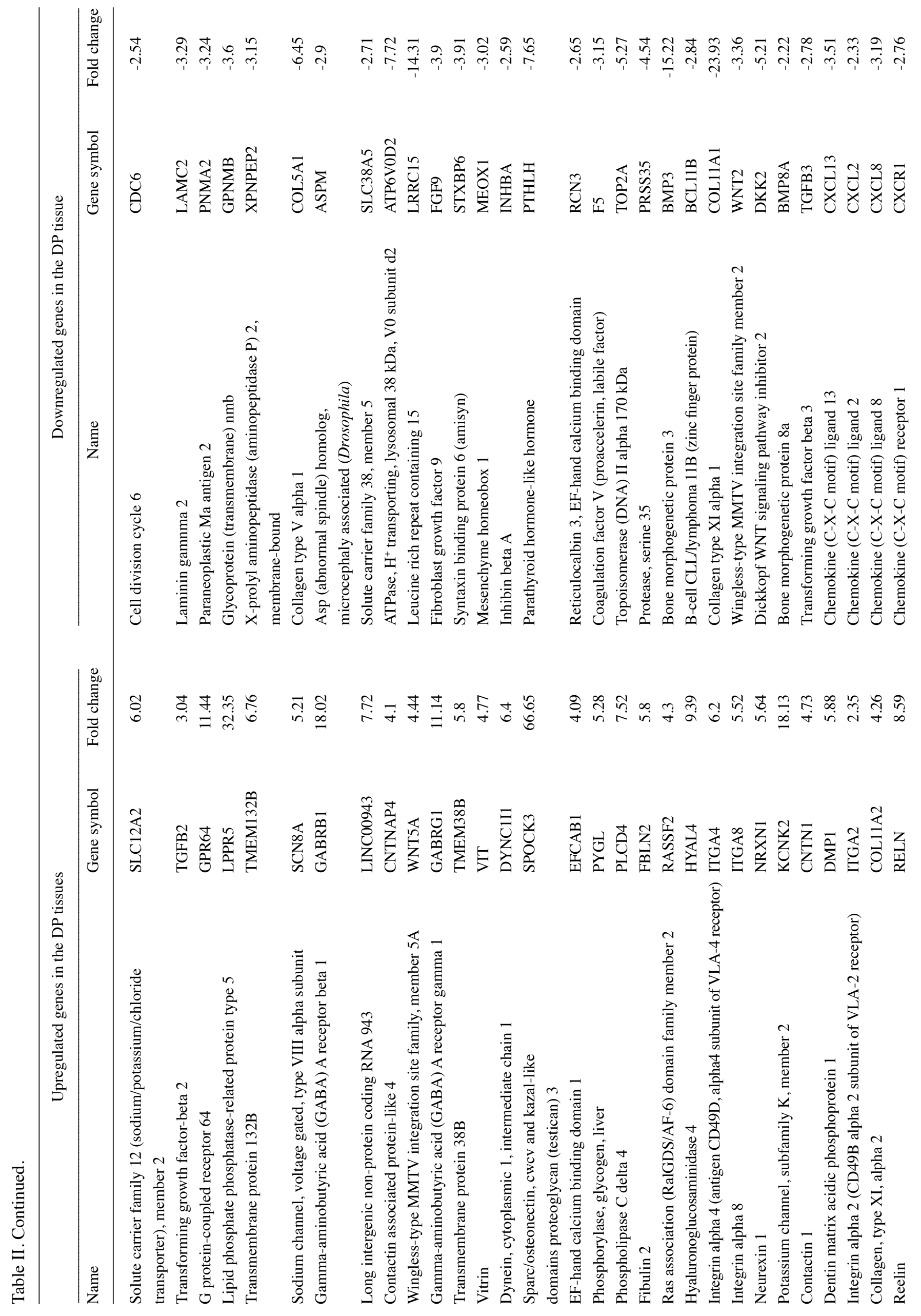




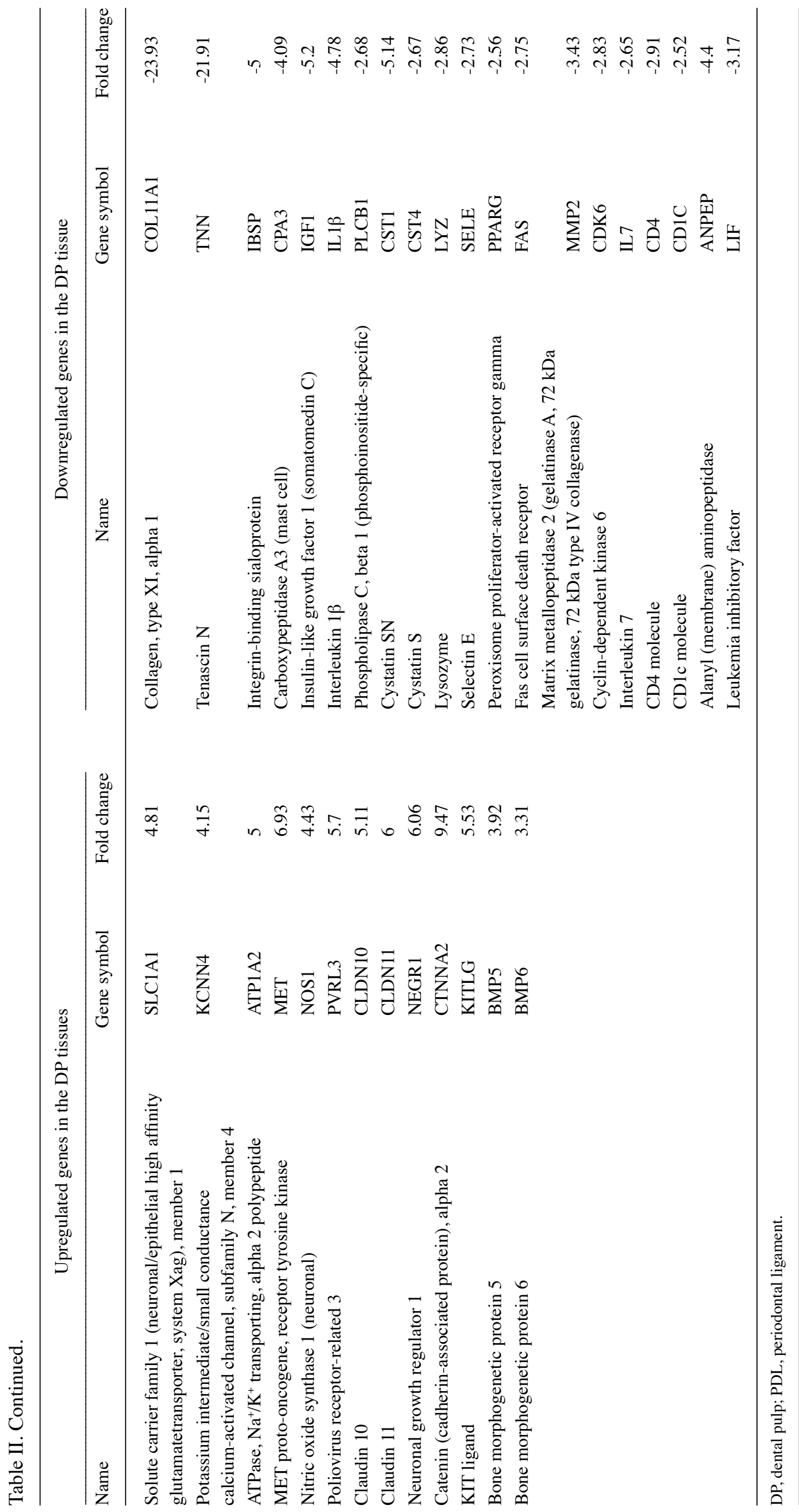




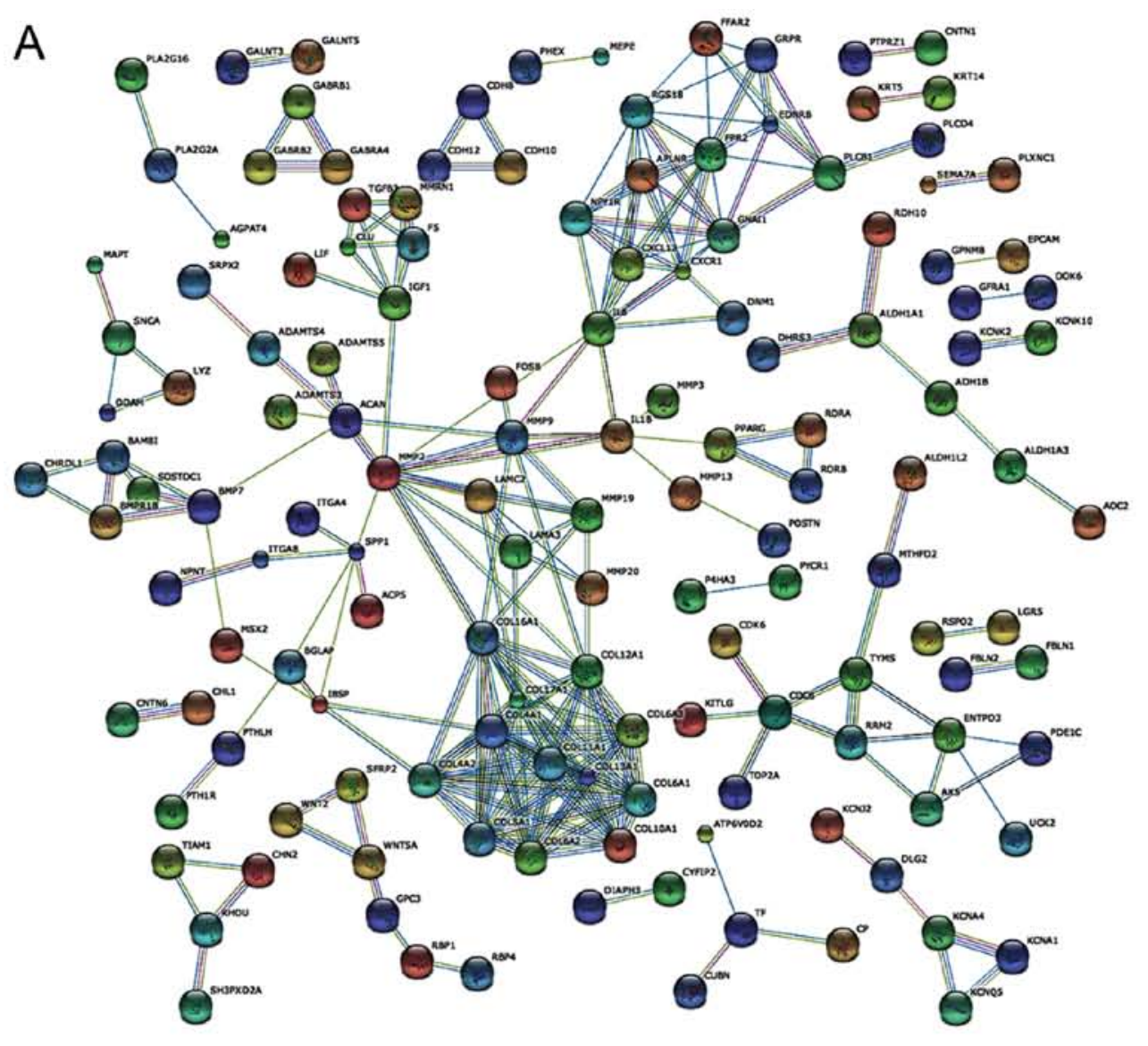

B ECM.RECEPTOR INTERACTION

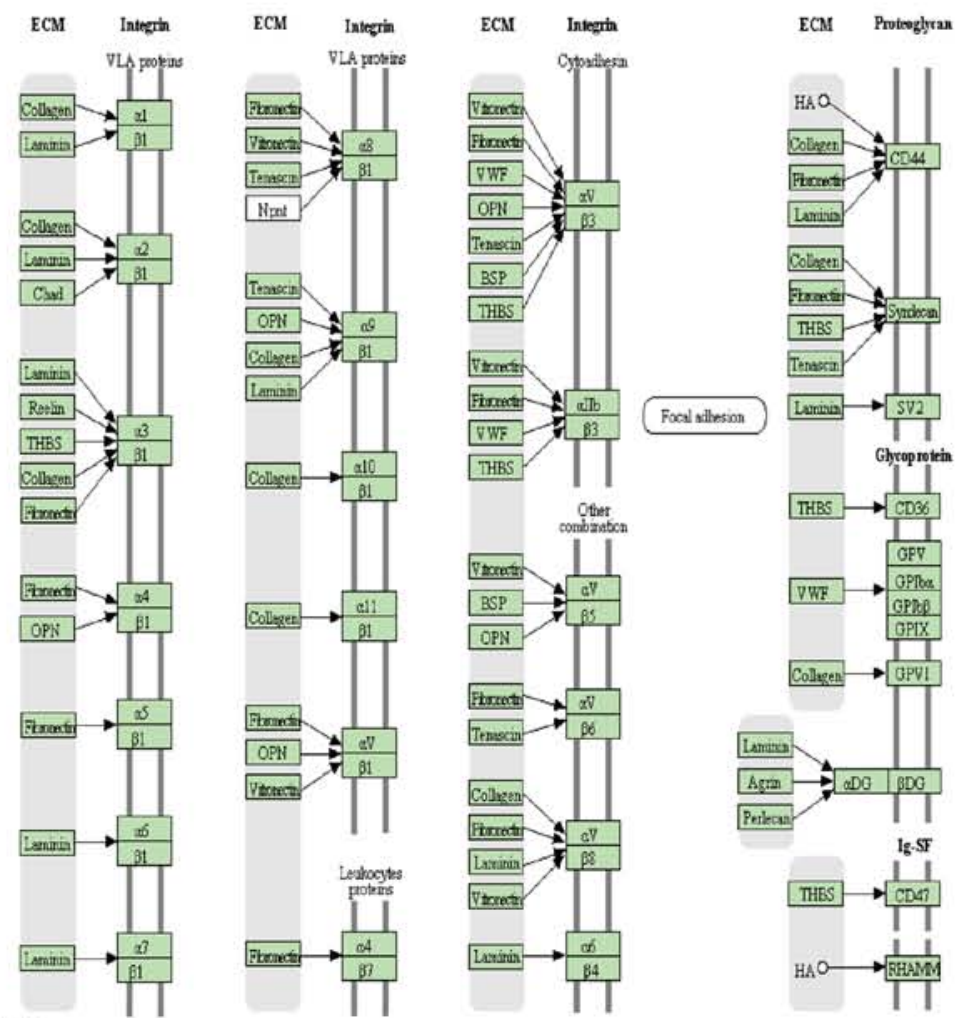


Table III. List of enriched KEGG pathways of the differentially expressed proteins.

\begin{tabular}{|c|c|c|c|}
\hline KEGG pathway & $\begin{array}{l}\text { No. of } \\
\text { hits }\end{array}$ & Expressed gene participating in the pathway & $\begin{array}{l}\text { Statistics for the enrichment } \\
\text { of the pathway }\end{array}$ \\
\hline $\begin{array}{l}\text { ECM-receptor } \\
\text { interaction }\end{array}$ & 18 & $\begin{array}{l}\text { COL11A2, ITGA8, ITGA4, RELN, COL11A1, COL6A3, } \\
\text { SPP1, CHAD, LAMA3, COL6A1, COL5A1, ITGA11, } \\
\text { COL4A1, TNN, LAMC2, IBSP, COL4A2, COL6A2 }\end{array}$ & $\begin{array}{l}\mathrm{C}=82 ; \mathrm{O}=18 ; \mathrm{E}=2.12 ; \mathrm{R}=8.47 \\
\text { raw } \mathrm{P}=2.55 \mathrm{e}-12 ; \operatorname{adj} \mathrm{P}=2.63 \mathrm{e}-10\end{array}$ \\
\hline $\begin{array}{l}\text { Protein digestion } \\
\text { and absorption }\end{array}$ & 16 & $\begin{array}{l}\text { COL6A2, COL17A1, COL4A2, COL11A2, CPA3, COL11A1, } \\
\text { SLC1A1, COL12A1, COL6A3, KCNN4, COL6A1, COL5A1, } \\
\text { DPP4, XPNPEP2, COL4A1, ATP1A2 }\end{array}$ & $\begin{array}{l}\mathrm{C}=76 ; \mathrm{O}=16 ; \mathrm{E}=1.97 ; \mathrm{R}=8.12 \\
\text { raw } \mathrm{P}=8.33 \mathrm{e}-11 ; \operatorname{adj} \mathrm{P}=4.29 \mathrm{e}-09\end{array}$ \\
\hline Focal adhesion & 20 & $\begin{array}{l}\text { COL6A2, COL4A2, COL11A2, ITGA8, ITGA4, RELN, } \\
\text { COL11A1, COL6A3, SPP1, CHAD, LAMA3, COL6A1, } \\
\text { COL5A1, IGF1, ITGA11, COL4A1, TNN, MET, LAMC2, IBSP }\end{array}$ & $\begin{array}{l}\mathrm{C}=195 ; \mathrm{O}=20 ; \mathrm{E}=5.05 ; \mathrm{R}=3.96 \\
\text { rawP=1.80e-07; adjP=6.18e-06 }\end{array}$ \\
\hline Amoebiasis & 11 & $\begin{array}{l}\text { LAMA3, COL4A2, COL11A2, COL5A1, IL8, TGFB3, } \\
\text { COL4A1, COL11A1, PLCB1, IL1B, LAMC2 }\end{array}$ & $\begin{array}{l}\mathrm{C}=104 ; \mathrm{O}=11 ; \mathrm{E}=2.69 ; \mathrm{R}=4.08 \\
\text { rawP=8.03e-05; adjP=0.0021 }\end{array}$ \\
\hline Salivary secretion & 8 & NOS1, CST1, PLCB1, SLC12A2, ATP1A2, KCNN4, CST4, LYZ & $\begin{array}{l}\mathrm{C}=78 ; \mathrm{O}=8 ; \mathrm{E}=2.02 ; \mathrm{R}=3.96 \\
\operatorname{raw} \mathrm{P}=0.0009 ; \operatorname{adj} \mathrm{P}=0.0185\end{array}$ \\
\hline $\begin{array}{l}\text { Cell adhesion } \\
\text { molecules (CAMs) }\end{array}$ & 10 & $\begin{array}{l}\text { CD4, ITGA8, ITGA4, PVRL3, SELE, NRXN1, } \\
\text { CLDN10, CLDN11, NEGR1, CNTN1 }\end{array}$ & $\begin{array}{l}\mathrm{C}=126 ; \mathrm{O}=10 ; \mathrm{E}=3.26 ; \mathrm{R}=3.06 \\
\operatorname{rawP}=0.0016 ; \operatorname{adj} \mathrm{P}=0.0265\end{array}$ \\
\hline Pathways in cancer & 18 & $\begin{array}{l}\text { MMP9, FGF9, COL4A2, PPARG, WNT2, CTNNA2, WNT5A, } \\
\text { FAS, LAMA3, IL8, MMP2, IGF1, TGFB3, KITLG, } \\
\text { COL4A1, MET, LAMC2, CDK6 }\end{array}$ & $\begin{array}{l}\mathrm{C}=319 ; \mathrm{O}=18 ; \mathrm{E}=8.27 ; \mathrm{R}=2.18 \\
\text { rawP=0.0018; } \operatorname{adj} \mathrm{P}=0.0265\end{array}$ \\
\hline Hematopoietic cell lineage & 7 & IL7, KITLG, CD4, IL1B, ITGA4, CD1C, ANPEP & $\begin{array}{l}\mathrm{C}=80 ; \mathrm{O}=7 ; \mathrm{E}=2.07 ; \mathrm{R}=3.38 \\
\text { raw } \mathrm{P}=0.0047 ; \operatorname{adj} \mathrm{P}=0.0543\end{array}$ \\
\hline $\begin{array}{l}\text { Cytokine-cytokine } \\
\text { receptor interaction }\end{array}$ & 14 & $\begin{array}{l}\text { IL7, LIF, CXCL13, BMP7, TNFRSF21, IL8, TGFB3, KITLG, } \\
\text { MET, IL1B, CXCR1, INHBA, BMPR1B, FAS }\end{array}$ & $\begin{array}{l}\mathrm{C}=248 ; \mathrm{O}=14 ; \mathrm{E}=6.43 ; \mathrm{R}=2.18 \\
\text { rawP=0.0054; } \operatorname{adj} \mathrm{P}=0.0543\end{array}$ \\
\hline Rheumatoid arthritis & 7 & ATP6V0D2, MMP3, IL1B, CTSK, IL8, ACP5, TGFB3 & $\begin{array}{l}\mathrm{C}=82 ; \mathrm{O}=7 ; \mathrm{E}=2.12 ; \mathrm{R}=3.29 \\
\operatorname{rawP}=0.0054 ; \operatorname{adj} \mathrm{P}=0.0543\end{array}$ \\
\hline
\end{tabular}

KEGG, Kyoto Encyclopedia of Genes and Genomes; ECM, extracellular matrix.

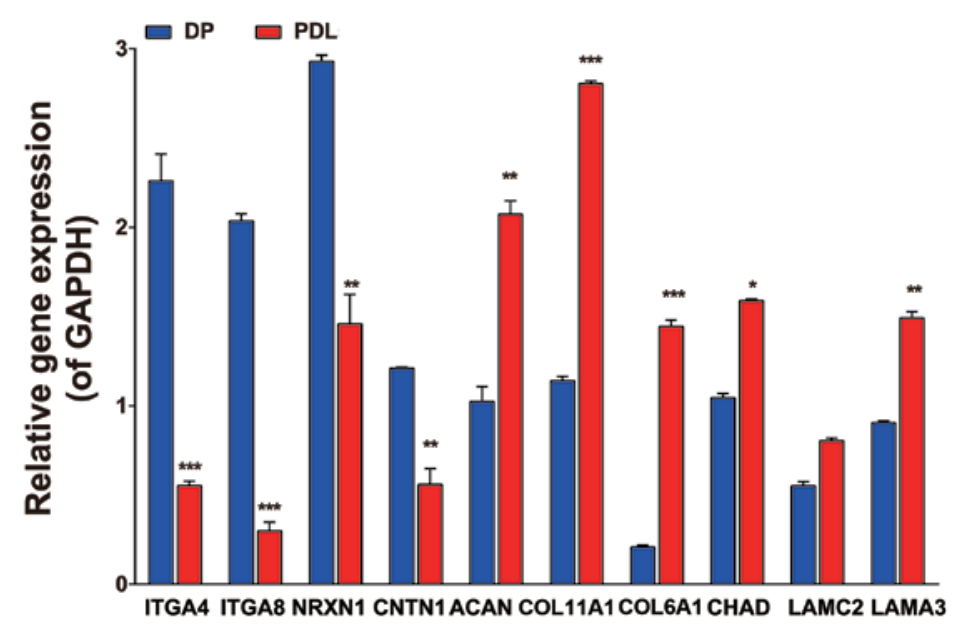

Figure 4. RT-qPCR of the relative difference in the mRNA expression extracted from dental pulp (DP) and periodontal ligament (PDL) tissues. mRNA expression levels of integrin alpha 4 (ITGA4), integrin alpha 8 (ITGA8) neurexin 1 (NRXN1), contactin 1 (CNTN1), aggrecan (ACAN), collagen type XI alpha 1 (COL11A1), collagen type VI alpha 3 (COL6A1), chondroadherin (CHAD), laminin gamma 2 (LAMC2) and laminin alpha 3 (LAMA3) were detected. "P<0.05, ${ }^{* *} \mathrm{P}<0.01$ and ${ }^{* * * *} \mathrm{P}<0.001$.

490 out of 33,297 (1.49\%) genes were differentially expressed between dental follicle and PDL tissues. This discrepancy may arise from the relative heterogeneity between DP and PDL tissues. 
To further analyze the DEGs, functional enrichment analyses were conducted using the WebGestalt tool. Our results revealed that the DEGs were associated with ECM-receptor interaction, protein digestion and absorption and focal adhesion, which were the three most enriched terms. The most enriched term ECM-receptor interaction is a complex network of different combinations of collagens, proteoglycans, hyaluronic acid, laminin, fibronectin and many other glycoproteins, including proteolytic enzymes involved in the degradation and remodeling of the ECM. Dentin ECM proteins play important roles in the dynamics of dentinogenesis $(24,25)$. Detailed studies of dentin matrix proteins may give some insights into unelucidated mechanisms of dentinogenesis.

Focal adhesion formation is initiated upon the binding of adhesion receptors to ECM ligands. The cell adhesion molecule, EpCAM, CNTN1 and NRXN1 had a higher expression in the DP tissues, while CHAD, LAMC2 and LAMA3 were upregulated in the PDL tissues. A recent study presented a close interaction of EpCAM with other cell-cell contact molecules, such as E-cadherin and claudins (26). CNTN1, a prototypical member of the contactin (CNTN) family, is involved, through cis- and trans-interactions with specific cell adhesion molecules, in neural cell migration, axon guidance and the organization of myelin subdomains (27). NRXN functions as synaptic transmission and maturation of contacts $(28,29)$. CHAD, a leucine rich repeat ECM protein with functions in cell to matrix interactions, is associated with both cartilage and bone homeostasis (30). Laminin, one of the major glycoprotein components, may maintain and regulate odontoblast differentiation and enamel crystallization (31).

As the ECM receptors in focal adhesions, integrins are heterodimeric transmembrane proteins that connect the actin cytoskeleton to the extracellular microenvironment and bidirectionally mediate signals across cell membrane $(32,33)$. ITGA2, ITGA4 and ITGA8 were upregulated in the DP compared with the PDL tissues. It has been have found that ITGA 2 is positively expressed in DP cells (34) and ITGA4 was stained in the dental neural crest-derived progenitor cells (dNC-PCs) (35). Adherent cells can identify laminin through ITGA2 for adhesion and differentiation (36), and recognize fibronectin via ITGA4 for attachment and migration (37). However, ITGA11 was upregulated in the DPL compared with the PD tissues. A previous study demonstrated that the ITGA11 knockout mice were characterized by a disorganized PDL, which may be attributable to the disturbed matrix metallopeptidase synthesis, and greatly reduced cell adhesion and spreading on collagen I (38). The regulation of matrix metalloproteinase-13 (MMP-13) and cathepsin K is ITGA11-dependent, which is involved in the coordinated extracellular and intracellular collagen proteolysis (39). In addition, integrin-binding sialoprotein (IBSP), which is a major structural protein of the bone matrix (40), was found to be upregulated in the PDL compared with the DP tissue in this study.

Some of the genes that were relatively strongly expressed in PDL tissues were associated with the degradation of the ECM. MMPs, the major players in collagen breakdown, have been identified in periodontal inflammation (41). The robust expressions of collagenases cathepsin K (CTSK) and MMP-3, -9 and -19, which were higher in the PDL tissue than in the DP tissue, are likely to play a part in the turnover of ECM in normal or pathological processes (41-43). A disintegrin and metalloproteinase with thrombospondin motif (ADAMTS14), which is zinc-dependent metalloproteinase and a member of the ADAMTS family of extracellular proteases, is involved connective tissue remodeling and inflammation (44). PDL cells may play a role in both the production and degradation of versican through the secretion of ADAMTS1, ADAMTS4 and ADAMTS5 (45).

In this study, the Wnt pathway member, Wnt family member 2 (WNT2), and Dickkopf-related protein 2 (DKK2), as well as the transforming growth factor (TGF) $\beta /$ bone morphogenetic protein (BMP) pathway member, BMP3, BMP8A and TGF $\beta 3$ were upregulated in the PDL tissues. Cells in the periodontal complex are Wnt responsive, and removing an important member of the Wnt signaling network gives rise to a pathological widening of the PDL space (46). However, other BMP family members (BMP7, BMP6 and BMP5) were upregulated in the DP tissues.

Of note, the PDL expressed more genes associated with inflammation or immune reaction than did the DP tissues. For example, CXCL2, 8 and 13, which are associated with chemotaxis, were upregulated in the PDL tissues. CXCL13, constitutively expressed in secondary lymphoid tissue, is a potent lymphoid chemokine (47). Studies have shown that CXCL13 is associated with B-cell recruitment in chronic inflammatory periodontal lesions (48). The results of this study suggest that anti-CXCL13 may be a promising approach to modulate pathogenic immune responses in PDL tissues.

Dentin sialophosphoprotein (DSPP), the most abundant non-collagenous protein in dentin, is a marker for DPSC differentiation into odontoblasts and is essential for the normal mineralization of dentin $(24,25,49,50)$. DSPP is processed by proteases into three primary domains: dentin sialoprotein (DSP), dentin phosphoprotein (DPP) and dentin glycoprotein (DGP). The dentin matrix proteins (DMPs) lead to tissue calcification due to inherent calcium binding properties in the ECM (50). Both DSPP and DMP1 were upregulated in the DP compared with the PDL tissue in this study.

Genes associated with osteogenic [osteopontin (OPN and osteocalcin $(\mathrm{OCN})$ ], osteoclastic [tartrate-resistant acid phosphatase (TRAP)] and chondrogenic (ACAN) functions were more strongly expressed in the PDL than in the DP tissue. OPN, indicated as Spp-1, is a multifunctional sialic acid-rich phosphorylated glycoprotein. OCN, shown as bone Gla protein, is a major non-collagenous protein. TRAP, known as ACP5, is involved in dissolugion of bone mineral though extracellular acidification (51). ACAN, one of the major components of the ECM, is mainly responsible for the high resistance to compression of the load-bearing tissue (52).

RT-qPCR analyses were performed to verify our cDNA microarray results. ITGA4, ITGA8, NRXN1 and CNTN were upregulated in the DP relative to the PDL tissue, while COL11A1, ACAN, COL6A1, CHAD, LAMC2 and LAMA3 were overexpressed in the PDL tissue compared with the DP tisue. These findings are in line with the microarray results.

In conclusion, this study compared gene expression profiles between DP and PDL tissues from human permanent teeth. Although only the RNA from the entire PD and PDL tissues was detected in this study, and not from the individual cell that constitutes these tissues, we consider that our results provide some novel insight into the characterization of DP and PDL 
tissues, and provide the potential molecular mechanisms concerning dental tissue mineralization and regeneration. The two types of tissue expressed specific genes related to their functions. The knowledge generated from this study demonstrated the differences between the DP and PDL tissues at the molecular biological level and may narrow down the field of potentially important signaling pathways for clinically relevant tissue regeneration.

\section{Acknowledgements}

We acknowledge financial support from the Nanjing Medical Science and Technology Development Project (grant no. YKK15133), and the Maternal and Child Healthcare Project of Jiangsu Province (grant no. F201557). This work was also funded by the National Natural Science Foundation of China (grant no. 81230022), the Priority Academic Program Development of Jiangsu Higher Education Institutions (grant no. PAPD-2014-37), the Natural Science Foundation of Jiangsu Province (grant nos. BL2014073 and 15KJA320002) and the Jiangsu Provincial Key Medical Discipline.

\section{References}

1. Chang CC, Chang KC, Tsai SJ, Chang HH and Lin $\mathrm{CP}$. Neurogenic differentiation of dental pulp stem cells to neuronlike cells in dopaminergic and motor neuronal inductive media. J Formos Med Assoc 113: 956-965, 2014.

2. Volponi AA, Pang Y and Sharpe PT: Stem cell-based biological tooth repair and regeneration. Trends Cell Biol 20: 715-722, 2010

3. Ledesma-MartínezE,Mendoza-Núñez VMand Santiago-OsorioE: Mesenchymal stem cells derived from dental pulp: A review. Stem Cells Int 2016: 4709572, 2016.

4. McCulloch CA: Proteomics for the periodontium: Current strategies and future promise. Periodontol 2000 40: 173-183, 2006.

5. Kim SH, Kim YS, Lee SY, Kim KH, Lee YM, Kim WK and Lee YK: Gene expression profile in mesenchymal stem cells derived from dental tissues and bone marrow. J Periodontal Implant Sci 41: 192-200, 2011.

6. Mrozik KM, Zilm PS, Bagley CJ, Hack S, Hoffmann P, Gronthos S and Bartold PM: Proteomic characterization of mesenchymal stem cell-like populations derived from ovine periodontal ligament, dental pulp, and bone marrow: Analysis of differentially expressed proteins. Stem Cells Dev 19: 1485-1499, 2010.

7. Eleuterio E,Trubiani O,SulpizioM,Di Giuseppe F,PierdomenicoL, Marchisio M, Giancola R, Giammaria G, Miscia S, Caputi S, et al: Proteome of human stem cells from periodontal ligament and dental pulp. PLoS One 8: e71101, 2013.

8. Gronthos S, Mankani M, Brahim J, Robey PG and Shi S: Postnatal human dental pulp stem cells (DPSCs) in vitro and in vivo. Proc Natl Acad Sci USA 97: 13625-13630, 2000.

9. Seo BM, Miura M, Gronthos S, Bartold PM, Batouli S, Brahim J, Young M, Robey PG, Wang CY and Shi S: Investigation of multipotent postnatal stem cells from human periodontal ligament. Lancet 364: 149-155, 2004.

10. Cha Y, Jeon M, Lee HS, Kim S, Kim SO, Lee JH and Song JS: Effects of in vitro osteogenic induction on in vivo tissue regeneration by dental pulp and periodontal ligament stem cells. J Endod 41: 1462-1468, 2015.

11. Yang ZH, Zhang XJ, Dang NN, Ma ZF, Xu L, Wu JJ, Sun YJ, Duan YZ, Lin Z and Jin Y: Apical tooth germ cell-conditioned medium enhances the differentiation of periodontal ligament stem cells into cementum/periodontal ligament-like tissues. J Periodontal Res 44: 199-210, 2009.

12. Mao JJ, Prockop DJ and Prockop DJ: Stem cells in the face: Tooth regeneration and beyond. Cell Stem Cell 11: 291-301, 2012

13. Han $X$ and Amar S: Identification of genes differentially expressed in cultured human periodontal ligament fibroblasts vs human gingival fibroblasts by DNA microarray analysis. J Dent Res 81: 399-405, 2002.
14. Lee HS, Lee J, Kim SO, Song JS, Lee JH and Lee SI, Jung HS and Choi BJ: Comparative gene-expression analysis of the dental follicle and periodontal ligament in humans. PLoS One 8: e84201, 2013.

15. Song JS, Hwang DH, Kim SO, Jeon M, Choi BJ, Jung HS, Moon SJ, Park W and Choi HJ: Comparative gene expression analysis of the human periodontal ligament in deciduous and permanent teeth. PLoS One 8: e61231, 2013.

16. Lee SW, Jeon M, Lee HS, Song JS, Son HK, Choi HJ, Jung HS, Moon SJ, Park W and Kim SO: Comparative gene-expression analysis of periodontal ligament and dental pulp in the human permanent teeth. J Korean Acad Pediatr Dent (JKAPD) 43: 166-175, 2016

17. McLachlan JL, Smith AJ, Bujalska IJ and Cooper PR: Gene expression profiling of pulpal tissue reveals the molecular complexity of dental caries. Biochim Biophys Acta 1741: 271-281, 2005.

18. Heikinheimo K, Kurppa KJ, Laiho A, Peltonen S, Berdal A, Bouattour A, Ruhin B, Catón J, Thesleff I, Leivo I, et al: Early dental epithelial transcription factors distinguish ameloblastoma from keratocystic odontogenic tumor. J Dent Res 94: 101-111, 2015.

19. Heikinheimo K, Jee KJ, Niini T, Aalto Y, Happonen RP, Leivo I and Knuutila S: Gene expression profiling of ameloblastoma and human tooth germ by means of a cDNA microarray. J Dent Res 81: 525-530, 2002.

20. Hochberg Y and Benjamini Y: More powerful procedures for multiple significance testing. Stat Med 9: 811-818, 1990.

21. Wang J, Duncan D, Shi Z and Zhang B: WEB-based GEne SeT AnaLysis Toolkit (WebGestalt): Update 2013. Nucleic Acids Res 41: W77-83, 2013.

22. Zhang B, Kirov S and Snoddy J: WebGestalt: An integrated system for exploring gene sets in various biological contexts. Nucleic Acids Res 33: W741-W748, 2005.

23. Franceschini A, Szklarczyk D, Frankild S, Kuhn M, Simonovic M, Roth A, Lin J, MinguezP, Bork P, von Mering C, et al: STRING v9.1: Protein-protein interaction networks, with increased coverage and integration. Nucleic Acids Res 41: D808-D815, 2013.

24. Butler WT, Brunn JC and Qin C: Dentin extracellular matrix (ECM) proteins: Comparison to bone ECM and contribution to dynamics of dentinogenesis. Connect Tissue Res 44 (Suppl 1): 171-178, 2003.

25. Butler WT: Dentin extracellular matrix and dentinogenesis. Oper Dent (Suppl 5): 18-23, 1992.

26. Martowicz A, Seeber A and Untergasser G: The role of EpCAM in physiology and pathology of the epithelium. Histol Histopathol 31: 349-355, 2016.

27. Mohebiany AN, Harroch S and Bouyain S: New insights into the roles of the contactin cell adhesion molecules in neural development. Adv Neurobiol 8: 165-194, 2014.

28. Missler M, Zhang W, Rohlmann A, Kattenstroth G, Hammer RE, Gottmann K and Südhof TC: Alpha-neurexins couple $\mathrm{Ca}^{2+}$ channels to synaptic vesicle exocytosis. Nature 423: 939-948, 2003.

29. Graf ER, Zhang X, Jin SX, Linhoff MW and Craig AM: Neurexins induce differentiation of GABA and glutamate postsynaptic specializations via neuroligins. Cell 119: 1013-1026, 2004.

30. Hessle L, Stordalen GA, Wenglén C, Petzold C, Tanner E, Brorson SH, Baekkevold ES, Onnerfjord P, Reinholt FP and Heinegård D: The skeletal phenotype of chondroadherin deficient mice. PLoS One 8: e63080, 2013.

31. Fukumoto $\mathrm{S}$ and Yamada Y: Review: Extracellular matrix regulates tooth morphogenesis. Connect Tissue Res 46: 220-226, 2005.

32. Iwamoto DV and Calderwood DA: Regulation of integrinmediated adhesions. Curr Opin Cell Biol 36: 41-47, 2015.

33. De Franceschi N, Hamidi H, Alanko J, Sahgal P and Ivaska J: Integrin traffic - the update. J Cell Sci 128: 839-852, 2015.

34. Zhu Q, Safavi KE and Spångberg LS: Integrin expression in human dental pulp cells and their role in cell attachment on extracellular matrix proteins. J Endod 24: 641-644, 1998.

35. Degistirici O, Jaquiery C, Schönebeck B, Siemonsmeier J, Götz W, Martin I and Thie M: Defining properties of neural crest-derived progenitor cells from the apex of human developing tooth. Tissue Eng Part A 14: 317-330, 2008.

36. Mercurio AM: Laminin receptors: Achieving specificity through cooperation. Trends Cell Biol 5: 419-423, 1995.

37. Aota $S$ and Yamada KM: Fibronectin and cell adhesion: Specificity of integrin-ligand interaction. Adv Enzymol Relat Areas Mol Biol 70: 1-21, 1995. 
38. Popova SN, Barczyk M, Tiger CF, Beertsen W, Zigrino P, Aszodi A, Miosge N, Forsberg E and Gullberg D: Alpha11 beta1 integrin-dependent regulation of periodontal ligament function in the erupting mouse incisor. Mol Cell Biol 27: 4306-4316, 2007.

39. Barczyk M, Bolstad AI and Gullberg D: Role of integrins in the periodontal ligament: Organizers and facilitators. Periodontol 2000 63: 29-47, 2013.

40. Chen J, McCulloch CA and Sodek J: Bone sialoprotein in developing porcine dental tissues: Cellular expression and comparison of tissue localization with osteopontin and osteonectin. Arch Oral Biol 38: 241-249, 1993.

41. Hannas AR, Pereira JC, Granjeiro JM and Tjäderhane L: The role of matrix metalloproteinases in the oral environment. Acta Odontol Scand 65: 1-13, 2007.

42. Beklen A, Al-Samadi A and Konttinen YT: Expression of cathepsin $\mathrm{K}$ in periodontitis and in gingival fibroblasts. Oral Dis 21: 163-169, 2015.

43. Sorsa T, Tjäderhane L, Konttinen YT, Lauhio A, Salo T, Lee HM, Golub LM, Brown DL and Mäntylä P: Matrix metalloproteinases: Contribution to pathogenesis, diagnosis and treatment of periodontal inflammation. Ann Med 38: 306-321, 2006.

44. Tang BL: ADAMTS: A novel family of extracellular matrix proteases. Int J Biochem Cell Biol 33: 33-44, 2001.

45. Sone S, Nakamura M, Maruya Y, Takahashi I, Mizoguchi I, Mayanagi $\mathrm{H}$ and Sasano Y: Expression of versican and ADAMTS during rat tooth eruption. J Mol Histol 36: 281-288, 2005.
46. Lim WH, Liu B, Cheng D, Williams BO, Mah SJ and Helms JA: Wnt signaling regulates homeostasis of the periodontal ligament. J Periodontal Res 49: 751-759, 2014.

47. Cyster JG, Ansel KM, Reif K, Ekland EH, Hyman PL, Tang HL, Luther SA and Ngo VN: Follicular stromal cells and lymphocyte homing to follicles. Immunol Rev 176: 181-193, 2000.

48. Nakajima T, Amanuma R, Ueki-Maruyama K, Oda T, Honda T, Ito $\mathrm{H}$ and Yamazaki K: CXCL13 expression and follicular dendritic cells in relation to B-cell infiltration in periodontal disease tissues. J Periodontal Res 43: 635-641, 2008.

49. Liu M, Sun Y, Liu Y, Yuan M, Zhang Z and Hu W: Modulation of the differentiation of dental pulp stem cells by different concentrations of $\beta$-glycerophosphate. Molecules 17: 1219-1232, 2012.

50. Ravindran S and George A: Dentin matrix proteins in bone tissue engineering. Adv Exp Med Biol 881: 129-142, 2015.

51. Karlström E,Ek-Rylander B, Wendel M and Andersson G: Isolation and phenotypic characterization of a multinucleated tartrateresistant acid phosphatase-positive bone marrow macrophage. Exp Hematol 39: 339-350.e3, 2011

52. Arner EC: Aggrecanase-mediated cartilage degradation. Curr Opin Pharmacol 2: 322-329, 2002. 